Breast Cancer Research and Treatment 18: 73-81, 1991.

(C) 1991 Kluwer Academic Publishers. Printed in the Netherlands.

Report

\title{
Multiple growth factor independence in rat mammary carcinoma cells
}

\author{
Stephen P. Ethier and Ramesh Moorthy \\ Department of Radiation Oncology, The University of Michigan Medical School, 1331 E. Ann St., \\ Ann Arbor, MI 48109, USA
}

Key words: autocrine factors, cholera toxin, epidermal growth factor, growth factor independence, insulin, rat mammary tumors

\begin{abstract}
In previous studies we demonstrated that rat mammary tumor (RMT) cells that are serially transplantable consist of cells that are independent of growth factors strictly required by normal rat mammary epithelial (RME) cells for growth in serum-free culture. The present studies were designed to determine the extent of the growth factor independence of several cell lines derived from these tumors and to determine if the cells that expressed growth factor independence in vitro are also tumorigenic in vivo. Cells from a transplantable mammary carcinoma (8-12 RMT) were seeded into culture in serum-free medium in the absence of either insulin (IN), epidermal growth factor (EGF), or cholera toxin (CT), and cell populations independent of the individual factors were developed. Next, the three growth factor independent populations were tested for their ability to grow in the absence of multiple growth factors. 8-12 RMT cells did not lose proliferative potential when multiple growth factors were deleted from the medium. Indeed, 8-12 RMT cells could be serially propagated in serum-free medium supplemented solely with bovine serum albumin (BSA) and ethanolamine. Cell lines independent of single growth factors were also developed from two other transplantable tumors (1-9 RMT and 7-15 RMT). In contrast to the 8-12 RMT-derived cell lines, deletion of additional growth factors from the media of the 1-9 RMT and 7-15 RMT-derived cells resulted in dramatic losses in growth potential. These results suggest that independence of individual growth factors is mediated by different mechanisms, since cells from different tumors can stably express independence of one, two, or three or more factors. Examination of conditioned media of four different RMT cell lines indicates that independence of EGF is mediated by autocrine factors. By contrast, there is no evidence for an autocrine factor that mediates independence of insulin-like growth factors. Thus, cell lines derived from serially transplantable RMTs are independent of either single or multiple growth factors, and independence of individual growth factors appears to be mediated by separate mechanisms.
\end{abstract}

\section{Introduction}

Normal cells, be they epithelial, fibroblastic, or hemopoietic, express strict requirements for spe- cific growth factors for proliferation in vitro [1-3]. Furthermore, the growth factor requirements of normal cells are complex in that multiple growth factor interactions are needed for growth stimula- 
tion $[4,5]$. For many cell types, the process of neoplastic transformation results in the acquisition of independence of specific growth factors required by normal cells, and there are many potential mechanisms of growth factor independence [6]. In some cell types, such as human melanoma cells, autocrine growth factor secretion mediates independence of specific factors. Normal human melanocytes require basic fibroblast growth factor for growth in culture [7], and human melanoma cells secrete this factor in a manner that relieves them of their requirement for the exogenous factor $[8,9]$. In other cell types, non-autocrine changes result in specific growth factor independence, such as in interleukin-3 dependent myeloid cells that are transformed to factor independence by an alteration in the c-abl proto-oncogene [10-12].

The work in our laboratory is aimed at understanding the growth factor requirements of normal rat mammary epithelial (RME) cells, characterizing the growth factor independence that occurs in these cells during neoplastic progession, and, ultimately, understanding at the cell and molecular level the alterations that mediate independence of specific factors. In previous studies, we characterized the growth factor requirements of normal RME cells [13] and demonstrated that rat mammary tumor (RMT) cells obtained from serially transplantable mammary carcinomas are independent of growth factors strictly required by normal cells for long-term growth in serum-free culture [14]. We have now developed a series of cell lines from these transplantable tumors that are defined on the basis of their ability to grow continuously in serum-free medium in the absence of specific growth factors. In order to study the cellular and molecular mechanisms of growth factor independence in these cells, it is necessary to first characterize these RMT cell lines with regard to the extent of the growth factor independence of each. Given that, the experiments presented in this report were designed to address four questions:

1) Do different growth factor independent cells express independence of multiple factors, or are RMT populations made up of sub-populations of cells independent of different factors?
2) Do different cell populations stably express different levels of growth factor independence?

3) Do autocrine factors play a role in mediating epidermal growth factor (EGF) independence of RMT cells?

4) Do RMT cells that are selected and maintained in culture on the basis of their growth factor independence retain neoplastic potential in vivo?

The results of all these experiments indicate that individual RMT cell populations can become independent of multiple growth factors and that independence of multiple growth factors is mediated by distinct alterations in growth control mechanisms. Furthermore, cells that express growth factor independence in vitro retain neoplastic potential in vivo.

\section{Materials and methods}

\section{Cell culture}

The methods for the serum-free culture of normal and neoplastic rat mammary epithelial cells have been published previously [13-15]. Briefly, cells are grown in hydroxethylpiperazine- $\mathrm{N}-2$ ethane sulfonic acid (HEPES)-buffered Ham's F-12 medium supplemented with insulin (IN), hydrocortisone (HC), EGF, cholera toxin $(\mathrm{CT})$, prolactin, progesterone, sodium selenite, triiodothyronine, ethanolamine, transferrin, and BSA. The cells are cultured on type-I collagen coated $60 \mathrm{~mm}$ tissue culture dishes. Normal RME cells and several cell lines developed in this laboratory from neoplastic rat mammary tissues were used in these studies. Cell lines designated 8-12 RMT, 7-15 RMT, and 1-9 RMT were derived from growth factor independent rat mammary carcinomas. The derivation of this culture medium, including analysis of the role and purity of BSA in this system, has been published previously [13], as has a detailed accounting of the influence of specific hormones and growth factors on the proliferative potential of RME and RMT cells $[14,15]$. 


\section{Deletion experiments}

To generate the growth factor independent cell populations, 8-12 RMT cells that had been prepared from serially transplanted tumors and had never been cultured were reactivated from frozen stocks and grown for one passage in fully growth factor supplemented medium containing $2 \%$ fetal bovine serum (FBS). At the first passage, the cells were divided into groups and cultured in serumfree medium either in the presence of a full complement of growth factors or in the absence of either IN, EGF, or CT. To compare the growth potential of the cells in the various media, cells were seeded at $10^{5}$ cells per $60 \mathrm{~mm}$ dish and grown for seven to ten days in these media. After the culture period, the number of cells per dish was determined by counting isolated nuclei with a Coulter Counter as described previously [14]. The cells that proliferated in the absence of individual growth factors were cultured in their respective single deletion media for five passages and prepared for transplantation (described below). At passage six, cells were tested for their ability to grow in the absence of multiple growth factors. Cells cultured in the absence of IN were seeded into $60 \mathrm{~mm}$ dishes and tested for their ability to grow in the additional absence of either EGF alone or EGF and CT. The cells were grown for seven to ten days, and the relative growth in the various media was compared by determining the increase in cell number as described above. Similar experiments were performed with cells derived by culture in the absence of EGF. Cells that expressed good growth potential in the absence of all three factors were maintained in triple deletion media for at least ten passages and then either transplanted or tested for their ability to grow in the absence of additional factors.

\section{Examination of conditioned medium for growth factor activity}

To determine if conditioned medium obtained from cultures proliferating in the absence of EGF contained EGF-like biological activity, experiments were performed using an indicator cell line that is growth factor dependent in serum-free medium. MCF-10 (kindly provided by $\mathrm{H}$. Soule and S. Brooks of the Michigan Cancer Foundation, Detroit, MI) [16] is a human mammary epithelial cell line derived from normal mammary epithelial cells that is absolutely dependent on EGF for growth in vitro. For these experiments, growth factor independent cells were grown to near confluence and conditioned medium was collected forty eighthours after the last feeding. The conditioned medium was centrifuged at $1000 \times \mathrm{g}$ for $10 \mathrm{~min}$ and tested for its ability to replace EGF for growth of MCF-10 cells. Indicator cells were seeded at $10^{5}$ cells per $60 \mathrm{~mm}$ dish and grown either in the presence of EGF or in EGF-free medium in the presence or absence of conditioned medium $(50 \% \mathrm{v} / \mathrm{v})$. The cells were grown for seven days and growth in the various media was compared as described above.

\section{Transplantation of RMT cells}

Cells to be transplanted were grown to confluence, harvested by trypsinization, and transplanted into the interscapular fat pads of syngeneic female Lew rats. Approximately $10^{6}$ cells were transplanted per fat pad, which for the non-cultured tumor cells is sufficient to yield tumors in $100 \%$ of host animals within four to six weeks.

\section{Materials}

IN, HC, EGF, prolactin, progesterone, triiodothyronine, sodium selenite, ethanolamine, hydroxyethylpiperazine-N-2 ethane sulfonic acid, and transferrin were obtained from Sigma Chemical Co. (St. Louis, MO), CT was from Schwartz-Mann (Spring Valley, NY), Vitrogen was from The Collagen Corporation (Palo Alto CA), and Ham's F-12 was from Hazelton Biologicals (Lenexa, KS). Lew rats were obtained from Charles River Breeding Laboratories (Kingston, NY). 


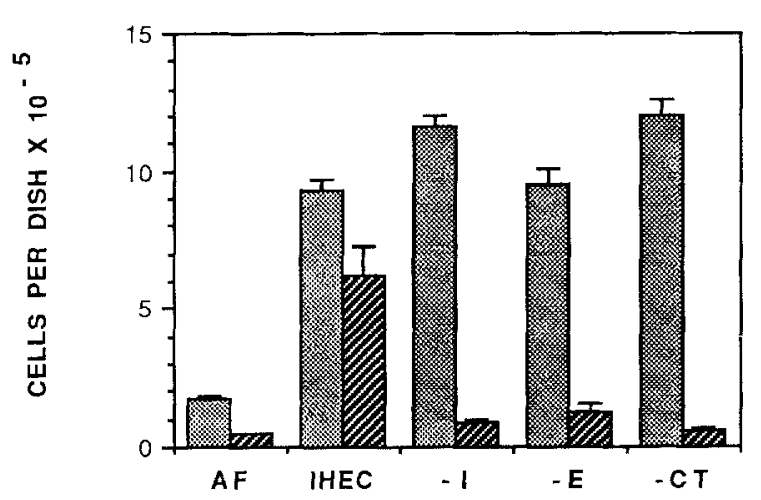

Fig. 1. Effect of deletion of individual growth factors on proliferation of 8-12 RMT (grey bars) and RME (hatched bars) cells in serum-free culture. Cells were seeded at $5 \times 10^{5}$ cells per $60 \mathrm{~mm}$ culture dish and grown for 10 days in the presence of insulin, hydrocortisone, epidermal growth factor, and cholera toxin (IHEC), or in the absence of individual factors. AF = attachment frequency; the number of attached cells one day after plating. Results are the mean number of cells per dish \pm the range for three plates per group at the end of the culture period. Stipled bars: $8-12$ RMT.

\section{Results}

\section{Multiple factor independence}

Normal RME cells require a complex culture medium to grow in the absence of serum (see Materials and methods). Of all of the supplements in this medium, the combination of IN, HC, EGF, and CT (or other CAMP agonists) is absolutely required for growth. Deletion of any of these factors results in a dramatic loss of growth potential.

Initial experiments with RMT cells were aimed at obtaining cultures in media devoid of either IN, EGF, or CT. The data in Fig. 1 show the comparative response of 8-12 RMT cells and normal RME cells to deletion of individual growth factors. The data show that 8-12 RMT cells do not lose proliferative potential when grown in the absence of either IN, EGF, or CT, whereas normal RME cells do not proliferate when any one factor is deleted.

To determine if $8-12$ RMT cells were truly independent of multiple factors, or if tumors consisted of multiple populations of cells that were independent of individual factors, a series of double and triple deletion experiments were performed with cells selected for at least 5 passages in media devoid

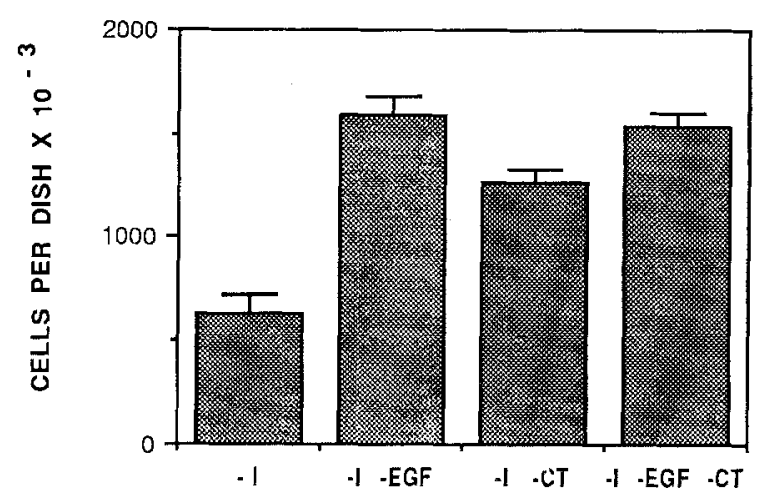

Fig. 2. Effect of deletion of multiple growth factors on the growth of IN-independent 8-12 RMT cells. 8-12 RMT cells were grown for at least 5 passages in the absence of IN. Cells were then subcultured and plated at $10^{5}$ cells per $60 \mathrm{~mm}$ culture dish and grown for seven days in medium devoid of single or multiple growth factors. Results are the mean number of cells per dish \pm the range for three plates per group at the end of the culture period.

of either IN or EGF. Figure 2 shows the results of such an experiment performed with cells selected for IN independence. The results indicate that further growth factor deletion did not retard the growth of these cells. Furthermore, cell growth was substantially better in the triple deletion media in which IN, EGF, and CT were absent than in the IN-free medium in which the cells were derived. The data in Fig. 3 show virtually identical results obtained with cells selected on the basis of EGF independence. These results indicate that 8 12 RMT cells consist of a population of cells that have lost the requirement for multiple factors for serum-free growth.

In subsequent experiments, additional factors that are important for RME cell growth in serumfree medium were deleted to determine the minimal requirements for growth of 8-12 RMT cells. As is shown in Fig. 4, 8-12 RMT cells growing in the absence of IN, EGF, and CT grew equally well upon deletion of hydrocortisone and transferrin. However, deletion of ethanolamine from the medium resulted in a rapid loss of growth potential. Thus 8-12 RMT cells can be grown and serially passaged in Ham's F-12 medium supplemented only with bovine serum albumin and ethanola- 


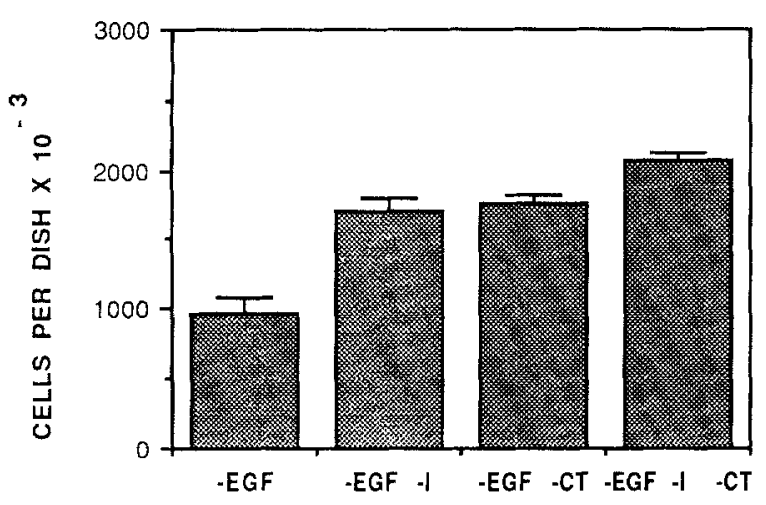

Fig. 3. Effect of deletion of multiple growth factors on the growth of EGF-independent 8-12 RMT cells. 8-12 RMT cells were grown for at least 5 passages in the absence of EGF. Cells were then subcultured and plated at $10^{5}$ cells per $60 \mathrm{~mm}$ culture dish and grown for seven days in medium devoid of single or multiple growth factors. Results are the mean number of cells per dish \pm the range for three plates per group at the end of the culture period.

mine. These cells have now been serially subcultured in this medium for twenty-five passages without any loss of proliferative capacity.

\section{Single factor independence}

The results of experiments performed with 812 RMT cells indicate that these cells are independent of multiple factors. To begin to determine if multiple growth factor independence is the result of multiple independent changes that take place during neoplastic progression, experiments were performed with growth factor independent cell strains derived from other serially transplantable tumors. One cell strain, designated 1-9 RMT-IN, is completely independent of IN for growth in serumfree medium. These cells grow to confluence over a seven day culture period in IN-free medium; however, deletion of either EGF or CT resulted in loss of growth potential such that no significant increase in cell number occurred over the same growth period in these media (Fig. 5a). Similar results were obtained with other lines that expressed independence of a single growth factor. 7-15 RMT-IN cells are also IN-independent, and whereas these cells have maintained their dependence on exogenous

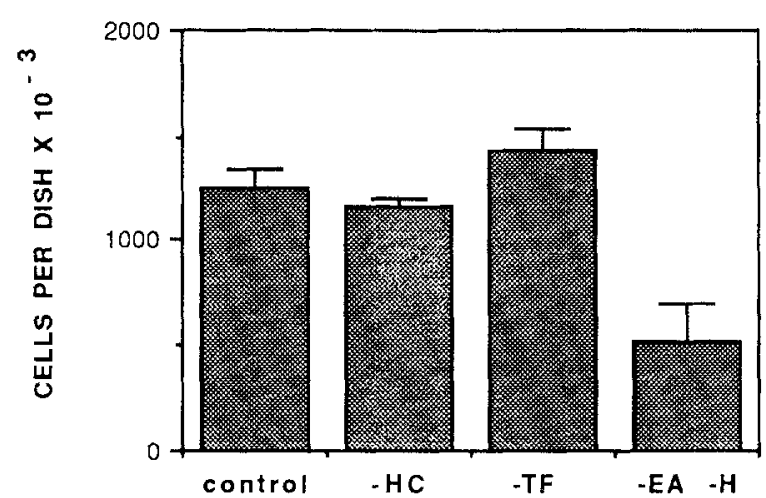

Fig. 4. Effect of deletion of additional medium supplements on growth of 8-12 RMT cells grown for several passages in the absence of EGF, IN, and CT. Triple deletion cells (control) were subcultured and seeded at $10^{5}$ cells per $60 \mathrm{~mm}$ dish and grown for seven days in triple deletion media or in triple deletion media in the additional absence of $\mathrm{HC}$, transferrin (TF), or ethanolamine and HEPES (EA, H). (Since HEPES is only required in this medium in the presence of the strongly basic ethanolamine, both agents are either added to or deleted from the medium together.) Results are the mean number of cells per dish \pm the range for three plates per group at the end of the culture period.

$\mathrm{EGF}$, they no longer require $\mathrm{CT}$ stimulation for growth in serum-free medium (Fig. 5b). Finally, 7-15 RMT-EGF cells are independent of EGF only and express an absolute requirement for IN and CT for growth in serum-free medium (Fig. 5c). Thus, the difference between multiple growth factor independent cells and cells independent of single factors is striking. These results suggest that acquisition of independence of individual growth factors occurs as a result of independent events, since certain cell lines consistently express independence of one, two, or three or more growth factors.

\section{Autocrine factors and independence}

Experiments have also been performed aimed at elucidating the mechanisms by which cells acquire independence of individual growth factors. The results of these studies are consistent with the hypothesis that cells become independent of insulinlike growth factors and EGF-like factors by distinct mechanisms. This difference becomes most apparent when one tests the autocrine hypothesis as it 

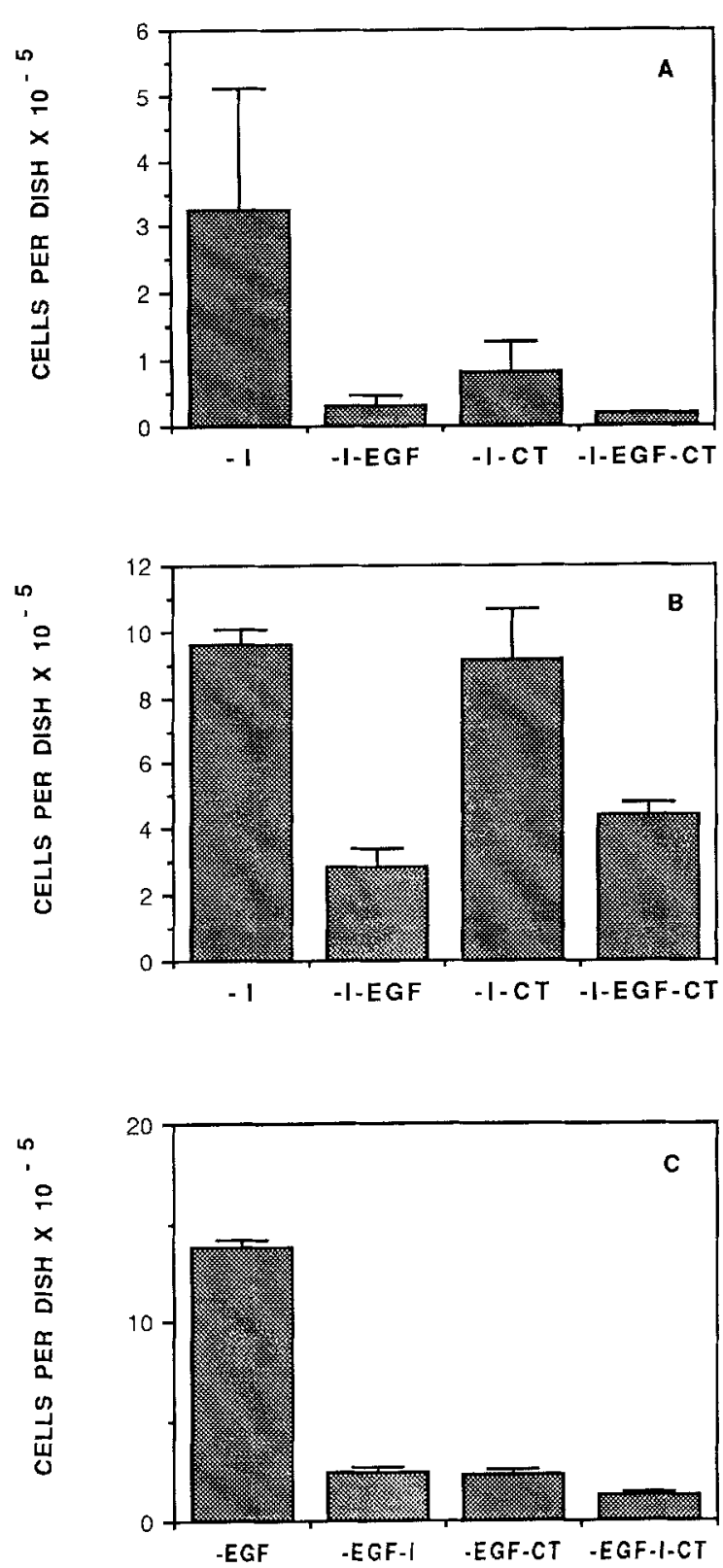

Fig. 5. Effect of multiple growth factor deletion on proliferative potential of 1-9 RMT and 7-15 RMT cells. Cells from these tumors were selected on the basis of the ability to grow in serum-free medium in the absence of individual growth factors and then tested for growth following deletion of multiple growth factors. a) 1-9 RMT-IN cells; b) 7-15 RMT-IN cells; c) 715 RMT-EGF cells. Experiments were performed as described in Figs 3 and 4.

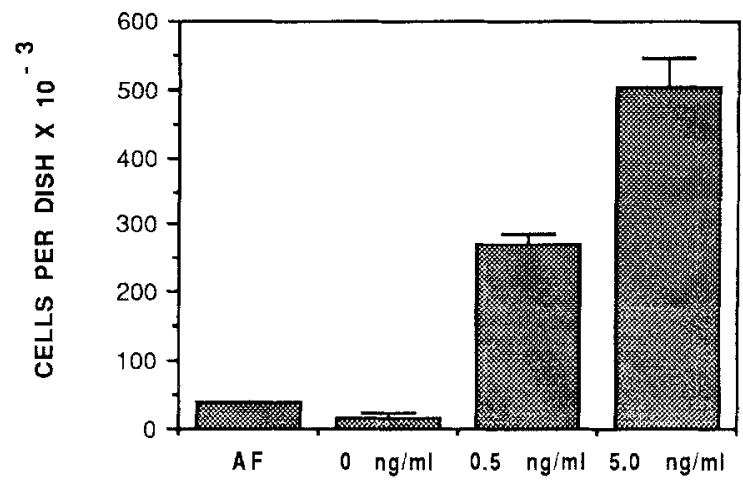

Fig. 6. Influence of EGF on growth of MCF-10 cells in serumfree medium. Cells were seeded at $5 \times 10^{4}$ per $35 \mathrm{~mm}$ culture dish and grown for seven days in serum-free medium, without EGF or in the presence of either $0.5 \mathrm{ng} / \mathrm{ml}$ or $5.0 \mathrm{ng} / \mathrm{ml}$ of EGF. Results are the mean number of cells per dish for three dishes \pm the range. $A F=$ the attached fraction: the number of attached cells per dish 24 hours after plating.

relates to growth factor independence of RMT cells. We have recently reported that there is no evidence for an autocrine growth factor that mediates IN independence of RMT cells [17]. By contrast, EGF independence does appear to be mediated by an autocrine loop.

Table 1 gives the results of experiments with four independently derived EGF-independent RMT cell lines. In these experiments, conditioned media obtained from cells that had been grown continuously in the absence of EGF were tested for their ability to replace EGF in a biological assay using MCF-10 cells as indicators. MCF-10 is a newly described human mammary epithelial cell line [15] that grows well in our serum-free medium and expresses an absolute requirement for both IN and EGF. The data in Fig. 6 shows the influence of EGF on the growth of MCF-10 cells in our serumfree medium. In the absence of EGF, there is no increase in cell number over a seven day culture period. By contrast, addition of 0.5 or $5.0 \mathrm{ng} / \mathrm{ml}$ EGF results in dramatic growth stimulation. Indeed, detectable EGF responses can be observed with these cells in the presence of as little as $0.1 \mathrm{ng} /$ $\mathrm{ml} \mathrm{EGF}$. In addition, this bioassay is performed in the presence of saturating concentrations of IN, hence IGF requirements are fulfilled. Under these conditions, MCF-10 cells do not respond to acidic 
Table 1. EGF-like bioactivity in RMT-derived conditioned medium. Conditioned medium was obtained from RMT cells that had been grown continuously in serum-free medium in the absence of EGF. The conditioned medium was tested for its ability to substitute for EGF in supporting the growth of MCF-10 cells in serum-free medium. Results given are the mean number of cells per dish for triplicate dishes of MCF-10 cells grown in the absence of EGF and in the presence or absence of conditioned medium from the indicated cell lines

\begin{tabular}{lcccc}
\hline & $8-12$ RMT-EGF & 1-9 RMT-EGF & 7-15 RMT-EGF & $6-17$ RMT-EGF \\
\hline Cells/dish & & & & 31,387 \\
- EGF & 7,247 & 17,677 & 31,387 & 214,107 \\
- EGF + CM & 59,573 & 68,420 & 105,580 & 6.8 \\
Fold increase & 8.2 & 3.9 & 3.4 & \\
\hline
\end{tabular}

fibroblast growth factor, basic fibroblast growth factor, or platelet derived growth factor (not shown). Thus, this bioassay is both sensitive and specific for EGF-like bioactivity. The data in Table 1 are from experiments in which MCF-10 cells were grown in the absence of EGF (IN is present in all cases) and in the presence or absence of conditioned medium obtained from EGF-independent cell strains. In all cases, conditioned media contained activity that could substitute for EGF for growth of MCF-10 cells. These results are consistent with the presence of an autocrine loop that mediates the EGF independence of the RMT cells. The factor responsible for the growth stimulating activity detected in the bioassay is negative in immunoassays for transforming growth factor-alpha and does not compete with ${ }^{125}$ I-EGF for binding to EGF receptors on MCF-10 cells (not shown). A more detailed accounting of this putative autocrine factor will be presented elsewhere.
In vivo growth potential of growth factor independent RMT cells

Previous experiments demonstrated a strict correlation between growth factor independence in vitro and serial transplantability in vivo [15]. To determine if cells selected and maintained in culture on the basis of their growth factor independence retained tumor potential in vivo, a series of growth factor independent 8-12 RMT cells were transplanted into syngeneic female rats. 8-12 RMT cells were reactivated from frozen stocks and selected for growth in media devoid of either IN, EGF, or CT. Cells were grown for at least five passages in the appropriate medium and harvested, and $10^{6}$ cells were transplanted into the interscapular fat pads of syngeneic host animals. With cells from the non-cultured tumor cell population, transplantation in this way yields tumors in $100 \%$ of the hosts within four to six weeks. The tumor potential of both non-cultured and cultured 8-12 RMT cells is shown in Table 2. All cell populations transplanted gave rise to tumors in every host animal.

Table 2. In vivo growth potential of RMT cells. RMT cells were transplanted into the interscapular white fat pads of syngeneic female Lew rats. $10^{6}$ cells per fat pad were transplanted and animals were monitored for six months. Tumors that did develop were palpable between one and two months after transplantation

\begin{tabular}{llll}
\hline Cell designation & Culture conditions & Tumor frequency & Tumor histology \\
\hline 8-12 RMT & Non-cultured & $6 / 6$ & Ductal CA \\
8-12 RMT & SF-Med. 5 pass. & $6 / 6$ & Ductal CA \\
8-12 RMT & - IN Med. 5 pass. & $3 / 3$ & Undiff. CA \\
8-12 RMT & - EGF Med. 5 pass. & $3 / 3$ & Undiff. CA \\
8-12 RMT & - CT Med. 5 pass. & $3 / 3$ & Undiff. CA
\end{tabular}

a $8-12$ RMT cells that are serially transplantable and have been maintained for three transplant generations and have not been cultured. b $8-12$ RMT cells cultured for 5 passages in serum-free medium in the presence of a full complement of growth factors (SF-Med). 


\section{Discussion}

It is now widely accepted that cancer cells have less stringent requirements for growth than do normal cells. However, interpretation of changes detected in cancer cells is limited by the level of understanding of normal cellular growth control mechanisms. The broad aim of our work is to study the proliferative potential of normal and neoplastic rat mammary epithelial cells using a culture system that supports the growth of both cell types. In this way, alterations in growth factor requirements that accompany neoplastic progression can be clearly defined and studied.

In previous work we demonstrated that in the rat mammary tumor model system, the acquisition of growth factor independence is associated with the acquisition of serial transplantability in vivo [15]. The previous results were correlative in nature. The results of the present experiments indicate that the cells that express growth factor independence in vitro, also express neoplastic potential in vivo. Furthermore, some RMT cells express long-term growth potential in media devoid of multiple factors that are required for growth of normal rat mammary epithelial cells. Whereas normal RME cells require a complex growth medium, 8-12 RMT cells no longer require IN, EGF, HC, cAMP agonists, or transferrin for growth in serum-free medium.

Given the extent of the difference in growth factor requirements between normal RME cells and serially transplantable $8-12$ RMT cells, it is unlikely that a single alteration mediates such large scale changes. Rather, it is more likely that cells undergo multiple changes that result in progressive acquisition of multiple growth factor independence. The data obtained thus far support this interpretation. 8-12 RMT cells are independent of multiple factors required by normal cells for growth in serum-free medium. This multiple growth factor independence is a characteristic of the entire population of 8-12 RMT cells, since in double and triple factor deletion experiments there was no loss of growth potential. If there were subpopulations of cells independent of multiple growth factors, one would have expected de- creased growth on a population basis with individual colonies emerging in the double and triple deletion media, and this was not observed. In contrast to the 8-12 RMT cells, however, cells from two other serially transplantable tumors expressed independence of one or two growth factors while retaining their requirements for other factors. These results indicate that a single alteration does not result in wholesale changes in growth factor requirements, but rather that independence of individual growth factors occurs by separate mechanisms. Finally, evidence currently available indicates that independence of insulin-like growth factors is not mediated by an autocrine factor [17], but that independence of EGF is mediated by an autocrine loop. Taken together, these results suggest that rat mammary carcinoma cells can become increasingly autonomous of exogenous factors for proliferation during the course of neoplastic progression in vivo.

These findings are relevant to results obtained with many other model systems. First, many types of epithelial cells of both rodent and human origin have been cultured in growth factor supplemented media. A common result that has emerged from this work is the requirement for IN-like growth factors, EGF, HC, and cAMP agonists that is shared by these diverse epithelial cell types [18-21]. Thus, these factors act in concert to stimulate a proliferative pathway that is common to many cell types. The acquisition of independence of these growth factors also appears to be a property common to many types of neoplastic cells. Several human cancer cell types can be cultured in a serumfree medium with little or no growth factor supplementation. In most of these cases, there is evidence for autocrine factor synthesis that stimulates in vitro growth [22-24]. However, as discussed above, normal cells have complex requirements for growth, and it is unlikely that synthesis of a single factor can mediate the changes that allow cells to grow in serum-free medium without any growth factor supplementation. In addition, there are numerous examples of cells that express growth factor independence without secretion of autocrine factors. In these cases, constitutive activation of oncoproteins with tyrosine kinase activity results in au- 
tonomy from exogenous growth factors $[10,11,25$, 26].

In summary, growth factor independent RMT cells have acquired autonomy of multiple factors for growth in serum-free culture, and these cells retain their tumor potential in vivo. Furthermore, the evidence suggests that more than one mechanism mediates the multiple growth factor independence observed in these cells, and that an autocrine loop is responsible for their EGF independence.

\section{Acknowledgements}

\section{Supported by Public Health Service grant CA 40064 from the NCI.}

\section{References}

1. Antoniades $\mathrm{HN}$, Albert AJ: Growth factors and regulation of cell growth. Ann Rev Med 33: 445-463, 1982

2. Heldin $\mathrm{CH}$, Westermark B: Growth factors: Mechanism of action and relation to oncogenes. Cell 37: 9-20, 1984

3. Metcalf $D$ : The roles of stem cell self-renewal and autocrine growth factor production in the biology of myeloid leukemia. Cancer Res 49: 2305-2311, 1989

4. Rozengurt E: Early signals in the mitogenic response. Science 234: 161-166, 1986

5. Sporn MB, Roberts AB: Peptide growth factors are multifunctional. Nature 332: 217-219, 1988

6. Sporn MB, Roberts AB: Autocrine growth factors and cancer. Nature 313, 745-747, 1985

7. Halaban R, Langdon R, Birchall N, Cuono C, Baird A, Scott G, Moellman G, McGuire J: Basic fibroblast growth factor from human keratinocytes is a natural mitogen for melanocytes. J Cell Biol 107: 1611-1619, 1988

8. Halaban R, Kwon BS, Ghosh S, Bovis PD: bFGF as an autocrine growth factor for human melanomas. Oncogene Res 3: 177-186, 1988

9. Sato $Y$, Rifkin DB: Autocrine activities of basic fibroblast growth factor: Regulation of endothelial cell movement, plasminogen activator synthesis, and DNA synthesis. J Cell Biol 107: 1199-1205, 1988

10. Cook WD, Metcalf D, Nicola NA, Burgess AW, Walker F: Malignant transformation of a growth factor-dependent myeloid cell line by Abelson virus without evidence of an autocrine mechanism. Cell 41: 677-683, 1985

11. Rovera $G$, Valtiere $M$, Mavilio F, Reddy EP: Effect of Abelson murine leukemia virus in granulocytic differentiation and interleukin-3 dependence of a murine progenitor cell line. Oncogene 1: 29-35, 1987
12. Daley GQ, Baltimore D: Transformation of an interleukin-3-dependent hemopoietic cell line by the chronic myelogenous leukemia specific P210bcr/abl protein. Proc Natl Acad Sci USA 85: 9312--9316, 1988

13. Ethier SP: Serum-free culture conditions for the growth of normal rat mammary epithelial cells in primary culture. In Vitro Cell Devel Biol 22: 485-490, 1986

14. Ethier SP, Kudla A, Cundiff KC: The influence of hormone and growth factor interactions on the proliferative potential of normal rat mammary epithelial cells in vitro. J Cell Physiol 132: 161-167, 1987

15. Ethier SP, Cundiff KC: Importance of extended growth potential and growth factor independence on in vivo neoplastic potential of primary rat mammary carcinoma cells. Cancer Res 47: 5316-5322, 1987

16. Soule HD, Maloney TM, Wolman SR, Peterson WD, Brenz R, McGrath CM, Russo J, Pauley RJ, Jones RF, Brooks SC: Isolation and characterization of a spontaneously immortalized human breast epithelial cell line, MCF-10. Cancer Res 50: 6075-6086, 1990

17. Ethier SP, Chiodino C, Jones RF: Role of growth factor synthesis in the acquisition of insulin/insulin-like growth factor I independence in rat mammary carcinoma cells. Cancer Res 50: 5351-5357, 1990

18. Babcock MS, Marino MR, Gunning WT, Stoner GD: Clonal growth and serial propagation of rat esophageal epithelial cells. In Vitro 19: 403-415, 1983

19. Bertolero F, Kaighn ME, Gonda MA, Safiotti U: Mouse epidermal keratinocytes: Clonal proliferation and response to hormones and growth factors in serum-free medium. Exp Cell Res 155: 64-80, 1984

20. Lechner JF, Jaugen A, McClendon IA, Pettis WE: Clonal growth of normal adult human bronchial epithelial cells in a serum-free medium. In Vitro 18: 633-642, 1982

21. Rozengurt E: Synergistic stimulation of DNA synthesis by cylic AMP derivatives and growth factors in mouse $3 \mathrm{~T} 3$ cells. J Cell Physiol 112: 243-250, 1982

22. El-Badry OM, Romanus JA, Helman LJ, Cooper MJ, Rechler MM, Israel MA: Autonomous growth of a human neuroblastoma cell line is mediated by insulin-like growth factor II. J Clin Invest 84: 829-839, 1989

23. Pichon F, Lagarde AE: Autoregulation of MeWo metastatic melanoma cell growth: Characterization of intracellu$\operatorname{lar}$ (FGF, MGSA) and secreted (PDGF) growth factors. J Cell Physiol 140: 344-358, 1989

24. Rodeck U, Herlyn M, Menssen HD, Furlanetto RW, Koprowski H: Metastatic but not primary melanoma cell lines grow independently of exogenous growth factors. Int J Cancer 40:687-690, 1987

25. Pierce JH, DiFiorre PP, Aaronson SA, Potter M, Pumphrey J, Scott A, Thle JN: Neoplastic transformation of mast cells by Abelson-MuL V: Abrogation of IL-3 dependence by a nonautocrine mechanism. Cell 41: 685-693, 1985

26. Wheeler EF, Rettenmier CW, Look AT, Sherr CJ: The $\mathrm{v}$-fms oncogene induces factor independence and tumorigenicity in CSF-1 dependent marcrophage cell line. Nature 324: $377-379,1986$ 